\title{
Enfermedad por reflujo gastroesofágico y enfermedades neurológicas en pediatría: complicaciones funcionales y motoras post funduplicatura de Nissen
}

\author{
María Alejandra Mortarini, Daniela Neder, Ana Rocca \\ Servicio de Gastroenterología, Hospital de Pediatría SAMIC "Prof. Dr. Juan P. Garrahan". Ciudad Autónoma de Buenos Aires, Argentina.
}

Acta Gastroenterol Latinoam 2020;50(4):453-458

Recibido: 09/04/2020 / Aceptado: 07//08/2020 / Publicado online: 14/12/2020

\section{Resumen}

La parálisis cerebral se asocia a alteraciones motoras. La prevalencia de enfermedad por reflujo gastroesofágico en niños con parálisis cerebral varia del $15 \%$ al $75 \%$. La funduplicatura de Nissen es la técnica antirreflujo más empleada, y no está exenta de complicaciones en esta población. Nuestro objetivo fue determinar la frecuencia y las caracteristicas de las complicaciones gastroenterológicas funcionales y motoras a largo plazo en pacientes neurológicos sometidos a funduplicatura de Nissen. Se realizó un estudio descriptivo de corte transversal, retrospectivo desde la recolección de datos, en pacientes de 0 a 18 años con compromiso neurológico, entre 2012 y 2017. Se incluyeron 35 pacientes. La mediana de

Correspondencia: María Alejandra Mortarini Combate de los Pozos 1881 (C 1245 AAM), Hospital "Prof. Dr. Juan P. Garrahan". Ciudad Autónoma de Buenos Aires, Argentina Tel.: +541141226229

Correo electrónico: alimortarini@gmail.com edad fue 55 meses (2-190). Se diagnosticó enfermedad por reflujo gastroesofágico en el 48\%; en el 52\% de estos el diagnóstico fue clinico. La funduplicatura fue laparoscópica en el $91,4 \%$ de los casos y se realizó gastrostomía en el mismo acto quirúrgico en el $86 \%$. En 25,7\% (n=9) hubo complicaciones funcionales y motoras: sindrome de Dumping (5,5\%), trastornos de la acomodación (11,4\%) y recidiva de reflujo (11,4\%). Fue necesario re-Nissen en el 8,5\%. En el 75\% de los pacientes con complicaciones se realizó gastrostomía en el mismo acto quirúrgico. No pudo demostrarse que la gastrostomía al momento de la cirugía antirreflujo se asociara a mayor frecuencia de complicaciones.

Palabras claves. Funduplicatura, reflujo gastroesofágico, parálisis cerebral.

\section{Long Term Functional Outcome after Nissen Fundoplication in Pediatric Population with Gastroesophageal Reflux and Cerebral Palsy}

\section{Summary}

Motility disorders are frequent in children with cerebral palsy. The prevalence of gastroesophageal reflux disease varies from the $15 \%$ to $75 \%$ in patients with cerebral palsy. Nissen fundoplication is the most used antireflux technique not without complications in this population. Our objective was to determine the frequency and the characteristics of the long-term functional and motility complications in patients with Nissen fundoplication and cerebral palsy. A descriptive 
cross-sectional study was performed. Data collection was acquired retrospectively with neurologic compromise between 2012 and 2017 in patients between 0 to 18 years old, with neurological impairment and Nissen fundoplication. $35 \mathrm{pa}$ tients were included. Median age was 55 months (2-190). Gastroesophageal reflux disease was diagnosed in the 48\%, in the $52 \%$ of this the diagnosis was clinical. It was performed by laparoscopic technique in the $91.4 \%$ of the cases gastrostomy was done in the same surgical time in $86 \%$ of the patients because of neurological impairment and risk of aspiration. In $25.7 \%(n=9)$ there were functional and motor complications, Dumping syndrome (5.5\%) accommodation disorders (11.4\%) and recurrence of reflux (11.4\%). Redo Nissen was required in the $8.5 \%$ of the patients $(n=3)$. In $75 \%$ of the patients with complications, gastrostomy was performed in the same surgical time. It could not be demonstrated that gastrostomy at the time of antireflux surgery was associated with greater frequency of complications.

Key words. Fundoplication, gastroesophageal reflux, cerebral palsy.

\section{Abreviaturas}

$R G E$ : Reflujo gastroesofágico.

ERGE: Enfermedad por reflujo gastroesofágico.

FN: Funduplicatura de Nissen.

VDG: Videodeglución.

SEGD: Seriada esófagogastroduodenal.

LA: Los Angeles (esofagitis).

VEDA: Videoendoscopía digestiva alta.

IBP: Inhibidores de la bomba de protones.

LAP: Laparoscopia.

GTT: Gastrostomía.

\section{Introducción}

El manejo del reflujo gastroesofágico (RGE) suele ser un tratamiento escalonado que se inicia con cambios higiénico-dietéticos y tratamiento supresor de la acidez gástrica enfocados al control de los síntomas, intentando reservar la indicación quirúrgica para casos refractarios. ${ }^{1}$

Las indicaciones más frecuentes de tratamiento quirúrgico se basan en el insuficiente control de los síntomas con el tratamiento médico, la necesidad de tomar medicación permanente, las manifestaciones respiratorias graves claramente relacionadas con reflujo, el esófago de Barrett, y en pacientes con enfermedad por reflujo (ERGE) y alteraciones neurológicas. ${ }^{1}$
Los objetivos del tratamiento quirúrgico consisten en la mejoría sintomática, curación de las lesiones mucosas, evitar complicaciones y reducir el uso de recursos sanitarios. ${ }^{1}$

La funduplicatura de Nissen (FN) es la técnica más empleada. No está exenta de complicaciones y morbilidad, especialmente en aquellos pacientes con patología neurológica y con estenosis. ${ }^{2}$ Las complicaciones quirúrgicas pueden ser: herniación del manguito, obstrucción de intestino delgado por adherencias, bridas, o drenajes intraperitoneales cuando está asociada a gastrostomía. La manipulación quirúrgica altera la función sensoriomotora y reduce el volumen gástrico disminuyendo la capacidad de acomodación. ${ }^{2}$ Se cree que sería responsable de complicaciones funcionales como incapacidad para eructar o vomitar cuando es necesario (síndrome de gas bloat), disfagia, síndrome de dumping por aceleración del vaciamiento gástrico y arcadas por retardo del mismo. ${ }^{2}$

La parálisis cerebral se asocia a menudo con alteraciones motoras que dificultan la alimentación por vía oral, por lo que frecuentemente se requiere la colocación de una gastrostomía como vía de alimentación. El reflujo en estos pacientes puede ser tratado con una cirugía adicional al mismo tiempo que la gastrostomía (con FN) o con medicación antirreflujo. Existen diferentes estudios que comparan el beneficio entre elegir uno u otro tratamiento. Hay revisiones sistemáticas que determinan cuál de los tratamientos puede ser más seguro y efectivo, si bien al momento no contamos con un estudio controlado aleatorizado. ${ }^{3}$

En pacientes con parálisis cerebral la ERGE se atribuye a la alteración de la motilidad del esófago y del esfínter esofágico inferior. La prevalencia de ERGE en estos pacientes varía del $15 \%$ al $77 \% .^{4}$ Los mecanismos fisiopatológicos son multifactoriales: la alteración neurológica puede causar un retraso en el vaciamiento gastrico y la motilidad esofágica. En estos pacientes la mayor prevalencia de ERGE también esta asociada al aumento de presion intraabdominal generado por las convulsiones, escoliosis, espasticidad de la musculatura abdominal y la constipación. ${ }^{5}$ Además de generar episodios de reflujo durante las relajaciones transitorias del esfínter esofágico inferior, se observa una alteración en la modulación de la inervación extrínseca relacionada con daño en el sistema nervioso central, causando disfunción motora esofágica. ${ }^{2}$ En un estudio, más del $75 \%$ de los cuidadores consideró que la FN mejoró la tolerancia a la alimentación y los vómitos, pero no se observó mejoría en los síntomas de dolor y disconfort al alimentarlos (arcadas, náuseas). Las complicaciones post FN referidas fueron: arcadas $20 \%$, dumping $3 \%$ y complicaciones quirúrgicas $10 \%{ }^{6}$ 
La necesidad de re-Nissen en los pacientes con compromiso neurológico es mayor $(15,4 \%)$ comparada con los pacientes sin alteración neurológica $(7 \%) .^{7}$ La causa más frecuente de re-Nissen es la herniación de la funduplicatura. La causa más frecuente de muerte es falla respiratoria por neumonía. Según lo publicado en una revisión de los factores de riesgo asociados a re-Nissen, el riesgo es significativamente más alto en aquellos pacientes con arcadas y edad temprana al momento de la funduplicatura. ${ }^{8}$

Inicialmente la bibliografía preconizaba el tratamiento quirúrgico combinado y precoz en estos niños, considerando que el daño neurológico permanente condicionaba un mal pronóstico del trastorno deglutorio y de la ERGE. La cirugía resultaba profiláctica en cuanto a preservar la función pulmonar de la micro o macroaspiración.

En los últimos años fue cambiando el paradigma. No solo se observó que la FN tenía peores resultados en estos niños y que además la causa del compromiso respiratorio era multifactorial, sino que además en algunos aparecían otros trastornos funcionales (dumping, trastornos de la acomodación, etc.), muy poco frecuentes en aquellos a los que se les ofrecía solo gastrostomía

El consenso europeo actual, publicado en 2017, en el que se realizaron recomendaciones para el manejo de pacientes con compromiso neurológico, sugiere una cuidadosa evaluación previo a realizar funduplicatura ya que los síntomas de ERGE en esta población de riesgo suelen ser silentes (los pacientes tienen dificultades en la comunicación), son inespecíficos (aumentan la espasticidad, convulsiones, manifestaciones respiratorias o laríngeas), o asociados a otras complicaciones (dificultades en la deglución o aspiración). Previo a la cirugía se requiere objetivar ERGE, buscar complicaciones (estenosis esofágica, Barrett) y descartar otros diagnósticos diferenciales (esofagitis eosinofílica). Sugieren realizar VEDA con biopsias previo a funduplicatura, estando indicado también realizar estudios contrastados, evaluar vaciamiento gástrico y realizar $\mathrm{pH}$ impedanciometría. Con respecto a la funduplicatura de Nissen, al momento de la colocación de gastrostomía sugieren que no se realice de rutina ya que aumenta la morbilidad. ${ }^{9}$

El objetivo de este trabajo es describir las complicaciones gastroenterológicas funcionales y motoras relacionadas a la FN y su tratamiento en pacientes con enfermedad neurológica.

La intención de elegir, dentro de todas las cirugías de Nissen que se realizaron (101), a niños con encefalopatía crónica no evolutiva fue homogeneizar la población. Es conocido que cuando hay factores genéticos, cirugías abdominales por otras causas o síndrome de Down, pueden sumarse factores que no harían fidedigna la muestra.

\section{Objetivos}

\section{Objetivo general}

- Determinar la frecuencia y las características de las complicaciones gastroenterológicas funcionales a largo plazo en pacientes neurológicos sometidos a funduplicatura de Nissen en un hospital pediátrico de alta complejidad.

\section{Objetivos específicos}

- Determinar la frecuencia de síntomas persistentes luego de la funduplicatura.

- Determinar las causas de re-Nissen.

- Determinar las complicaciones motoras posteriores a funduplicatura en pacientes con compromiso neurológico, tales como recidiva de reflujo, trastornos de acomodación, síndrome de dumping.

\section{Métodos}

Entre 2012 y 2017 se realizó un estudio descriptivo de corte transversal, retrospectivo desde la recolección de datos, en pacientes con compromiso neurológico de 0 a 18 años de edad, sometidos a funduplicatura de Nissen en un hospital pediátrico de alta complejidad (Hospital Garrahan). Se evaluaron las historias clínicas y se recolectó: edad al momento de la cirugía, antropometría, síntomas y tratamientos realizados previamente, estudios contrastados de tracto digestivo superior, monitoreo prolongado de RGE, valoración endoscópica y síntomas funcionales (trastornos de la acomodación y síndrome de dumping) y motores (recidiva de reflujo) posteriores a la cirugía. La colocación del electrodo de pHmetría o impedanciometría con pHmetría se realizó bajo control radioscópico. Se consideró patológico un índice de exposición ácida mayor a $6 \%$ en los mayores de un año y mayor a $12 \%$ en los menores de un año, número de episodios de reflujo mayor a 70 en mayores de un año y mayor a 100 en menores de un año, o un índice sintomático mayor o igual a 50\%. ${ }^{10}$

Se incluyeron pacientes menores de 18 años con compromiso neurológico a los que se realizó funduplicatura de Nissen como tratamiento de ERGE. Los criterios de exclusión fueron pacientes con otra cirugía abdominal, pacientes en quienes se realizó cirugía de Nissen en otro centro y pacientes con síndrome de Down.

Para la recolección de datos se utilizó una planilla del programa de Red Cap 8.9.2. Se aplicaron medidas de resumen y dispersión para el análisis de las variables continuas. Las variables categóricas se resumieron como proporciones o porcentaje. Se evaluó asociación estadística a través un análisis univariado con $\mathrm{chi}^{2}$. Se utilizó R Studio como programa estadístico. 


\section{Resultados}

En los últimos cinco años se realizaron en nuestro centro 101 funduplicaturas de Nissen, 35 de ellas en pacientes con encefalopatía crónica no evolutiva, los cuales fueron incluidos en este trabajo.

La mayoría de los pacientes eran de sexo masculino $(65,7 \%)$. Un 48\% tenía diagnóstico de reflujo gastroesofágico realizado por $\mathrm{pHmetría} \mathrm{con} \mathrm{o} \mathrm{sin} \mathrm{impedanciome-}$ tría y / o VEDA. En el 52\% de los pacientes el diagnóstico de reflujo fue clínico. El 91,4\% (32) recibió tratamiento para el mismo previo a la cirugía.

Con respecto a la cirugía, la mediana de edad fue de 55 meses (2-190). La mayoría se realizó por vía laparoscópica $91,4 \%$ (32), y en el 86\% (31) de los casos se realizó gastrostomía en el mismo acto quirúrgico. No se evidenció mortalidad relacionada a la cirugía en ninguno de los pacientes.

En 25,7\% (9) de los pacientes presentaron complicaciones funcionales y motoras posteriores a la cirugía de Nissen. Se observó en dos pacientes síndrome de dumping, cuatro pacientes con trastornos de la acomodación y cuatro con recidiva de reflujo (un paciente presentó dos complicaciones: síndrome de dumping y trastorno de la acomodación). El diagnóstico de síndrome de dumping se confirmó con prueba de tolerancia oral a la glucosa en uno de los pacientes, y en el otro la prueba arrojó un resultado dudoso, iniciándose tratamiento por la sospecha clínica. Recibieron como intervención primaria el agregado de almidón a la fórmula y fraccionamiento de esta con mejoría, sin necesidad de otra intervención.

Cuatro pacientes $(11,4 \%)$ presentaron trastornos en la acomodación, manifestados por arcadas como síntoma principal. Se realizó como intervención primaria el fraccionamiento de la alimentación, a lo cual respondió un solo paciente. Dos pacientes requirieron una segunda intervención con alimentación por goteo continuo con mejoría de los síntomas. Un paciente requirió inicio de ciproheptadina con resolución clínica.

Se observó recidiva de reflujo en cuatro pacientes $(11,4 \%)$, dos de ellos presentaron vómitos sin respuesta al reiniciar IBP, evidenciando válvula flap no continente por SEGD y VEDA. Dos pacientes presentaron hemorragia digestiva alta, con esofagitis severa reiniciando IBP. Tres pacientes requirieron re-Nissen $(8,5 \%)$. En todos los pacientes con complicaciones se había realizado funduplicatura LAP y en el $75 \%$ se había hecho una GTT en el mismo acto quirúrgico.

Ningún paciente tenía estudios de vaciamiento gástrico, manometría, como tampoco estudios de medicina nuclear para valorar aspiración.

El análisis univariado para evaluar asociación con complicaciones post funduplicatura no mostró una diferencia estadísticamente significativa.

\section{Discusión}

En los pacientes con enfermedad neurológica es frecuente la presencia de ERGE; el objetivo del tratamiento siempre es aliviar los síntomas con el menor número de efectos colaterales.

En una revisión sistemática realizada en el año 2013 no se encontró ningún ECCA que evaluara si la funduplicatura es mejor tratamiento antirreflujo que el tratamiento médico exclusivo. Por lo que concluye que se necesitan estudios comparativos para poder realizar una indicación y elegir el mejor tratamiento con sustento científico. ${ }^{3}$ En nuestro estudio el $74 \%$ de los pacientes presentó mejoría clínica (resolución de ERGE y ausencia de otros síntomas post FN), aunque desconocemos cuál hubiera sido la evolución del reflujo si se hubiera realizado solamente gastrostomía.

La funduplicatura de Nissen es el procedimiento quirúrgico más realizado para el tratamiento del reflujo gastroesofágico en niños. ${ }^{11} \mathrm{El}$ abordaje laparoscópico es una técnica efectiva y segura para el tratamiento del ERGE; actualmente es considerada la técnica de elección. En nuestro estudio, en la mayoría de los casos se realizó abordaje laparoscópico $(91,4 \%)$. Requirieron re-Nissen el $8,5 \%$, coincidente con lo publicado. ${ }^{7}$

Los síntomas frecuentemente referidos luego de la funduplicatura son: disfagia, incapacidad de eructar, saciedad precoz, bloating, dispepsia, síndrome de gas bloat, arcadas, dolor, rechazo a la alimentación, diarrea y síndrome de dumping. ${ }^{2}$ En nuestro estudio se observaron complicaciones funcionales y motoras en el $25,7 \%$ de los casos. En los pacientes con síndrome de dumping se observó resolución de los síntomas con el agregado de almidón en las fórmulas, suspendiendo hidratos de carbono simples y fraccionando la alimentación. Con respecto a los trastornos de acomodación se utilizó ciproheptadina en una paciente que no había tenido respuesta a fraccionar la alimentación y luego a goteo continuo. La misma fue efectiva, con mejoría clínica. En la bibliografía está descrita la utilidad de esta droga en los pacientes con arcadas post funduplicatura ya que mejoran la acomodación gástrica y/o la hipersensibilidad a la distensión que ocurre en estos casos. ${ }^{12}$

Dos pacientes presentaron recidiva de ERGE: un paciente por disrupción del manguito y otro por falla primaria, considerando re-Nissen en ambos casos.

$\mathrm{Al}$ recopilar los datos se evidencia que no contábamos con un estudio sistematizado de valoración previa a la funduplicatura en estos pacientes. Se objetivó RGE con $\mathrm{pH}$-metría o pH-impedanciometría en $80 \%$ pacientes, siendo patológica en el $53,5 \%$. Se conoce que la $\mathrm{pH}$-impedanciometría es la herramienta más sensible para eva- 
luar RGE en pacientes con síntomas típicos y atípicos. ${ }^{13}$ Un estudio evaluó con $\mathrm{pH}$-impedanciometría a pacientes pre y post funduplicatura, encontrando que el número total de episodios de reflujo y el número de episodios de reflujo proximales eran significativamente mayores en los pacientes con reflujo patológico que en los pacientes con RGE no patológico. Se observó mayor número de episodios de reflujo proximal en pacientes alimentados con sonda nasogástrica, presentando más episodios de reflujo no ácido comparado con aquellos pacientes alimentados vía oral. Por lo que se concluyó que la $\mathrm{pH}$-impedanciometría es una herramienta útil para detectar reflujo en pacientes neurológicos con reflujo no ácido, con pH-metría normal, o como primera instancia en aquellos pacientes con alta sospecha de reflujo y alteraciones neurológicas. ${ }^{14}$

En un metaanálisis realizado en China se compararon los estudios que incluían la funduplicatura laparoscópica versus la convencional, evaluándose tres ECCA (total 171 pacientes). ${ }^{15}$ No se encontraron diferencias estadísticamente significativas en cuanto a mortalidad, complicaciones postoperatorias, readmisiones relacionadas con complicaciones y la duración de la internación. Se observó que la vía laparoscópica se asocia con menor porcentaje de pacientes que presentan arcadas post funduplicatura, así como mayor recurrencia que en los pacientes con cirugía convencional, aunque con evidencia baja o muy baja. ${ }^{16}$ No se observó mortalidad relacionada con la cirugía en nuestro estudio.

Con respecto a realizar la funduplicatura de Nissen al momento de la realización de colocación de gastrostomía, sugieren que no se realice de rutina ya que aumenta la morbilidad. ${ }^{9}$ En nuestro estudio, a todos los pacientes con complicaciones se les había realizado funduplicatura LAP y en el 75\% se había hecho una GTT en el mismo acto quirúrgico.

Sin embargo, al evaluar asociación GTT durante el mismo acto quirúrgico y su relación con la presencia de complicaciones, el análisis no fue estadísticamente significativo.

La revisión de la bibliografía de los últimos años y la experiencia clínica nos motivó a revisar retrospectivamente que pasó con nuestra población, e intentar valorar exhaustivamente la presencia de reflujo gastroesofágico para no realizar cirugías que aumenten la morbimortalidad. Nuestro estudio presenta limitaciones, ya que al ser retrospectivo, no todos los pacientes tienen el mismo seguimiento e iguales estudios previos a la decisión de realizar funduplicatura. Asimismo, no se evaluó el grado de satisfacción de padres o cuidadores de los pacientes con relación a la cirugía a través de encuestas o scores, como ha sido referido en la bibliografía. ${ }^{17}$

\section{Conclusión}

En nuestra casuística, un $25,7 \%$ de los pacientes manifestó complicaciones funcionales y motoras luego de la cirugía de Nissen. Un 11,4\% de los pacientes presentó recidiva del RGE luego del tratamiento quirúrgico. No pudo demostrarse que la gastrostomía al momento de la cirugía antirreflujo se asociara a mayor frecuencia de complicaciones.

Conflicto de intereses. Los autores no presentan conflictos de intereses.

Sostén financiero. No se recibió financiamiento para este trabajo.

\section{Referencias}

1. Armas Ramos H, Ortigosa Castillo L. Reflujo gastroesofágico y esofagitis en niños. En: F. Argüelles Martín - M.D. García Novo, ed. Tratado de gastroenterología, hepatología y nutrición pediátrica aplicada de la SEGHNP2010 Ergon C/ Arboleda, 1. 28221 Majadahonda (Madrid) 2010: 163-176.

2. Nurko S. Gastric function after fundoplication. Pediatric Neurogastroenterology. En: Faure, Taphar, Di Lorenzo (eds.) Springer 2017: 343-347.

3. Vernon-Roberts A, Sullivan PB. Fundoplication versus postoperative medication for gastro-oesophageal reflux in children with neurological impairment undergoing gastrostomy [Internet]. Cochrane Database of Systematic Reviews 2013. Disponible en: http://dx.doi.org/10.1002/14651858.cd006151.pub3

4. Fernando T, Goldman RD. Management of gastroesophageal reflux disease in pediatric patients with cerebral palsy. Can Fam Physician 2019; 65 (11): 796-798.

5. Vandenplas Y, Hegar B. Diagnosis and treatment of gastro-oesophageal reflux disease in infants and children [Internet]. Journal of Gastroenterology and Hepatology 2000; 15: 593-603. Disponible en: http://dx.doi.org/10.1046/j.1440-1746.2000.02169.x(3)

6. O'Loughlin EV, Somerville H, Shun A, Gangemi R, La Hei E, Desphande A, O'Loughlin TF. Antireflux Surgery in Children With Neurological Impairment [Internet]. Journal of Pediatric Gastroenterology and Nutrition 2013; 56: 46-50. Disponible en: http://dx.doi.org/10.1097/mpg.0b013e318267c320

7. Martin K, Deshaies C, Emil S. Outcomes of Pediatric Laparoscopic Fundoplication: A Critical Review of the Literature [Internet]. Canadian Journal of Gastroenterology and Hepatology 2014; 28: 97-102. Disponible en: http://dx.doi.org/10.1155/2014/738203

8. Baerg J, Thorpe D, Bultron G, Vannix R, Knott EM, Gasior AC, Sharp SW, Tagge E, St. Peter SD. A multicenter study of the incidence and factors associated with redo Nissen fundoplication in children. J Pediatr Surg 2013; 48 (6): 1306-1311.

9. Romano C, Van Wynckel M, Hulst J, Broekaert I, Bronsky J, Dall'Oglio L, et al. European Society for Paediatric Gastroenterology, Hepatology and Nutrition Guidelines for the Evaluation and Treatment of Gastrointestinal and Nutritional Complications in Children With Neurological Impairment. J Pediatr Gastroenterol Nutr 2017; 65 (2): 242-264. 
10. Rudolph CD, Mazur LJ, Liptak GS, Baker RD, Boyle JT, Colletti RB, et al. Guidelines for evaluation and treatment of gastroesophageal reflux in infants and children: recommendations of the North American Society for Pediatric Gastroenterology and Nutrition. J Pediatr Gastroenterol Nutr 2001; 32 Suppl 2: S1-S31.

11. Rosen R, Vandenplas Y, Singendonk M, Cabana M, DiLorenzo C, Gottrand F, et al. Pediatric Gastroesophageal Reflux Clinical Practice Guidelines [Internet]. Journal of Pediatric Gastroenterology and Nutrition 2018; 66: 516-554. Disponible en: http:// dx.doi.org/10.1097/mpg.0000000000001889

12. Rodríguez L, Diaz J, Nurko S. Safety and efficacy of cyproheptadine for treating dyspeptic symptoms in children. J Pediatr 2013; 163 (1): 261-267.

13. Leung AKC, Hon KL. Gastroesophageal reflux in children: an updated review [Internet]. Drugs in Context 2019; 8: 1-12. Disponible en: http://dx.doi.org/10.7573/dic.212591
14. Fukahori S, Asagiri K, Ishii S, Tanaka Y, Kojima S-I, Saikusa N, et al. Pre and post-operative evaluation of gastroesophageal reflux and esophageal motility in neurologically impaired children using combined $\mathrm{pH}$-multichannel intraluminal impedance measurements. Pediatr Surg Int 2013; 29 (6): 545-551.

15. Zhang P, Tian J, Jing L, Wang Q, Tian J, Lun L. Laparoscopic vs. open Nissen's fundoplication for gastro-oesophageal reflux disease in children: A meta-analysis [Internet]. International Journal of Surgery. 2016; 34: 10-16. Disponible en: http://dx.doi. org/10.1016/j.ijsu.2016.08.017

16. Pascoe E, Falvey T, Jiwane A, Henry G, Krishnan U. Outcomes of fundoplication for paediatric gastroesophageal reflux disease. Pediatr Surg Int 2016; 32 (4): 353-361.

17. Ceriati E, Marchetti P, Caccamo R, Adorisio O, Rivosecchi F, De Peppo F. Nissen fundoplication and combined procedures to reduce recurrence of gastroesophageal reflux disease in neurologically impaired children. Sci Rep 2020; 10 (1): 11.618. 